\title{
Highly sensitive force measurements in an optically generated, harmonic hydrodynamic trap
}

\author{
Iliya D. Stoev ${ }^{1,2}$, Benjamin Seelbinder ${ }^{1,2}$, Elena Erben ${ }^{1,2}$, Nicola Maghelli ${ }^{1,2}$ and Moritz Kreysing ${ }^{1,2,3^{*}}$
}

\begin{abstract}
The use of optical tweezers to measure forces acting upon microscopic particles has revolutionised fields from material science to cell biology. However, despite optical control capabilities, this technology is highly constrained by the material properties of the probe, and its use may be limited due to concerns about the effect on biological processes. Here we present a novel, optically controlled trapping method based on light-induced hydrodynamic flows. Specifically, we leverage optical control capabilities to convert a translationally invariant topological defect of a flow field into an attractor for colloids in an effectively one-dimensional harmonic, yet freely rotatable system. Circumventing the need to stabilise particle dynamics along an unstable axis, this novel trap closely resembles the isotropic dynamics of optical tweezers. Using magnetic beads, we explicitly show the existence of a linear force-extension relationship that can be used to detect femtoNewton-range forces with sensitivity close to the thermal limit. Our force measurements remove the need for laser-particle contact, while also lifting material constraints, which renders them a particularly interesting tool for the life sciences and engineering.
\end{abstract}

Keywords: Force measurements, Optofluidics, Hydrodynamic trap, Thermoviscous flows

\section{Introduction}

We recently showed [1] how an optically generated flow field, which adaptively pushes a particle towards a target position, is sufficient to counteract the particle's diffusion on timescales longer than the feedback time. As the flow velocity at the position of the particle was independent from its radial displacement, we concluded that this hydrodynamic trap was not suitable for force measurements. Here we present a novel optically induced hydrodynamic trap that effectively relies on the passive self-centring of a particle in a quasi-1D flow field. This field is characterised by a linear force-displacement relationship that enables the measurement of external forces. To this end, we transitioned from a flow dipole

\footnotetext{
*Correspondence: kreysing@mpi-cbg.de

${ }^{1}$ Max Planck Institute of Molecular Cell Biology and Genetics,

Pfotenhauerstraße 108, 01307 Dresden, Germany

Full list of author information is available at the end of the article
}

to a quadrupole-like flow field making use of two opposing collinear thermoviscous flow fields $[2,3]$ by splitting the scanning line of an infrared laser into two counterdirected paths on the same axis (Fig. 1a). Technically, these are operated in a time-shared manner on a sub-millisecond timescale. Specifically, the direction of laser scanning dictates the direction of the induced flows and a stagnation point is formed between the two counterdirected laser scan paths (horizontal arrows in Fig. 1b) in a region without direct laser exposure. This excludes the possibility for optical trapping. For a particle situated on the axis between the two laser paths, the resulting flow field is effectively one-dimensional, with a positionally invariant stagnation point that represents a virtual attractor for the particle at all times. In contrast to an alternative design of hydrodynamic flow traps introduced by Tanyeri et al. [4, 5] that operates under the constraints of a given microfluidic chamber geometry, our system is characterised by a translationally invariant stagnation point that defines 
the centre of the trap, even in the case of particle displacements. The reduction to one dimension renders this new trap inherently stable and isotropic, and mitigates the need to stabilise particle dynamics along an extensional axis via the adaptive repositioning of this stagnation point.

Moreover, our optical approach neither requires the incorporation of intersecting microchannels nor that of sophisticated optically driven microrotors as in Būtaite et al. [6] but directly generates a complex hydrodynamic flow field inside a generic microscopy chamber consisting of two parallel glass surfaces. Furthermore, in a static flow field, any positional fluctuation along the perpendicular, extensional axis (vertical arrows in Fig. 1b) would result in the expulsion of the particle. To avoid this, we made use of the optical control capabilities of this microfluidic system. Specifically, we used active feedback control [1] that here enabled the dynamic rotation of the counterflows and rapid realignment of the compressional axis with the particle's extension, such as to effectively restore the 1D geometry (Fig. 1d).

\section{Results and discussion}

\subsection{Trapping confirmation}

Having first determined the effective viscosity increase due to proximity to the chamber wall (Additional file 4: Fig. S1), we performed repeated trapping experiments by switching the trap on and off sporadically (Fig. 2a). As soon as the laser was turned on, the particle was dragged towards the stagnation point, and when the laser was turned off, it diffused out again in a Brownian manner. Analysis revealed that the profile of the trapping potential (see positional histogram in Fig. 2b) is symmetric, confirming that the magnitude of the restoring force achieved by dynamic optical rotation of the laser scan paths (cf. Fig. 1d) is independent of the direction of particle displacement from the stagnation point. Our feedback-based optical control effectively creates a quasi1D trapping situation. Here, the particle appeared always to be displaced along the compressional axis only, circumventing the need to stabilise the particle motion in the perpendicular, intrinsically unstable direction. As an important consequence, the stagnation point always remained in the same position. Trapping into this translation-invariant stagnation point was further confirmed by analysis of the mean squared displacement (MSD) of a $3-\mu \mathrm{m}$ bead (Additional file 4: Fig. S2), indicating nearly identical trapping along both in-plane directions.

\subsection{First estimate of an apparent trap stiffness}

To obtain a first estimate for the strength of the particle confinement, we analysed the power spectral density (PSD) using a procedure for processing discrete positional data outlined elsewhere [7,8]. The transition between short and long time diffusion was marked in the PSD plot (Fig. 2c) by a corner frequency $f_{c}$, which at this point allowed the phenomenological estimation of the stiffness $k$ along each orthogonal axis via $f_{c}=k /\left(12 \pi^{2} \eta R\right)$. Using this approach, we obtained apparent trap stiffnesses of $35 \pm 3 \mathrm{fN} / \mu \mathrm{m}$ along the $\mathrm{x}$-axis and $41 \pm 4 \mathrm{fN} / \mu \mathrm{m}$ along the $\mathrm{y}$-axis. These values provide sensitivity that compares favourably to previously reported optical-tweezer estimates [7, 9, 10]. Thus, optically induced thermoviscous flows appear able to generate highly sensitive traps without the need of any direct laser exposure.

\subsection{Linear force-displacement relationship}

To utilise this optofluidic trap for force measurements, it is essential that the displacement from a trapping point can be used as a readout of the force, to which a particle is subjected. We therefore investigated the

(See figure on next page.)

Fig. 1 Force trap generated by two counterdirected thermoviscous flow fields that align with the direction of a particle's displacement from the central stagnation point. a Experimental setup: rapid laser scanning (red beam) induces counterdirected flows (blue arrows) on a common axis (termed 'compressional' or 'trapping' axis) with a probe particle, giving rise to a stagnation point. Along each scan path, the direction of the flows runs opposite to that of the laser scanning. The blue arrows point along the compressional axis, also shown with horizontal white arrows in panel b. $\mathbf{b}$ Visualisation of the generated flow-field lines and its central stagnation point (purple) using tracer beads (Additional file 1: Video SV1), with horizontal and vertical arrows indicating the trapping and extensional axes, respectively. c Linear fit to the velocity-displacement plot of tracer particles near the stagnation point in a stationary flow field. The shaded lightblue region encloses data from particles in contact with the laser scan paths. $\mathbf{d}$ Snapshots demonstrating the restoring character of the thermoviscous flows (white arrows). Depending on the direction of particle displacement $\delta r$ from the stagnation point, the flow field is automatically rotated (double-headed arrows) in a way that effectively counteracts this displacement (Additional file 2: Video SV2). Dotted lines indicate dynamically co-rotating trapping axis. e Rhodamine intensity profile (red arrows correspond to scan paths) and relative temperature increase upon application of the counterflows. The difference in the image intensity can be directly used to quantify the relative temperature increase as a function of laser driving current (LDC). The laser power we used throughout the experiments in this work corresponds to LDC of $900 \mathrm{~mA}$ and led to maximum heating along the scan paths of around $3 \mathrm{~K}$, given that the intensity of the dye changes by $1.3 \% / \mathrm{K}$. The inset shows the level of heating in the region around the stagnation point at each LDC, where the micromanipulated object is trapped (maximum heating of $0.5 \mathrm{~K}$ ). Error bars represent standard deviations, obtained from a total of 3 repeats for 700-800 mA and 2 repeats for 850-900 mA 
(a)

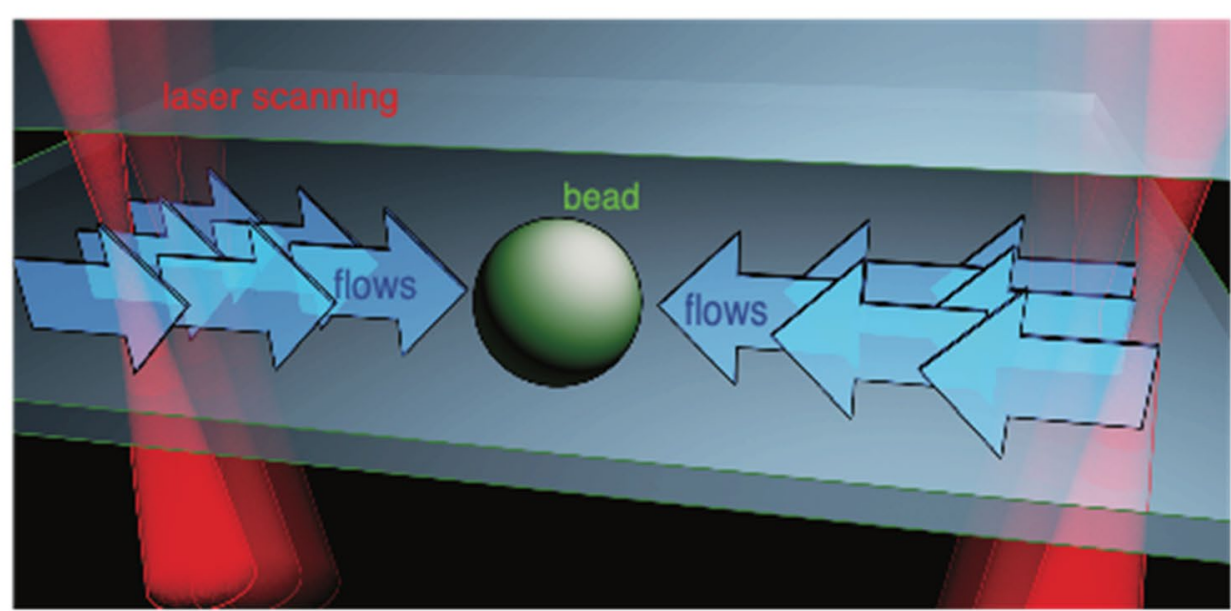

(b)

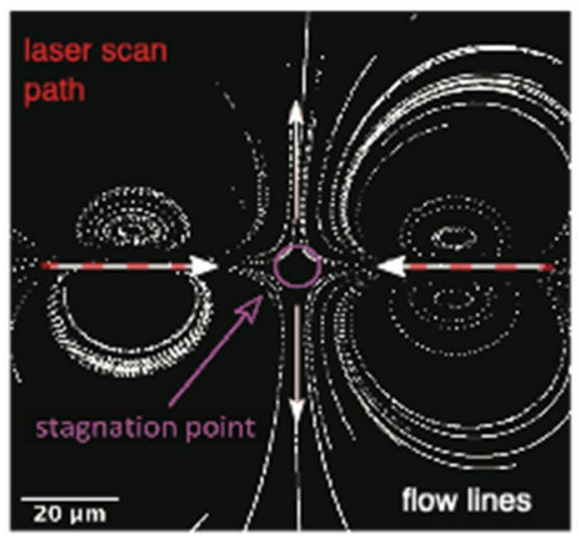

(d)
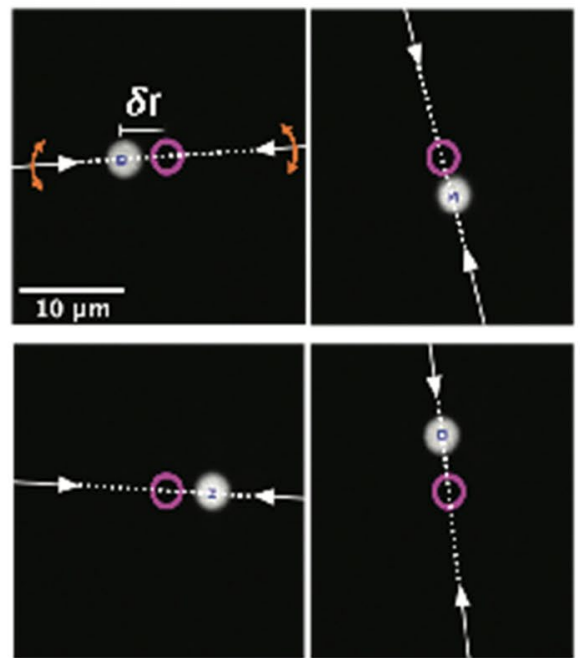

(c)

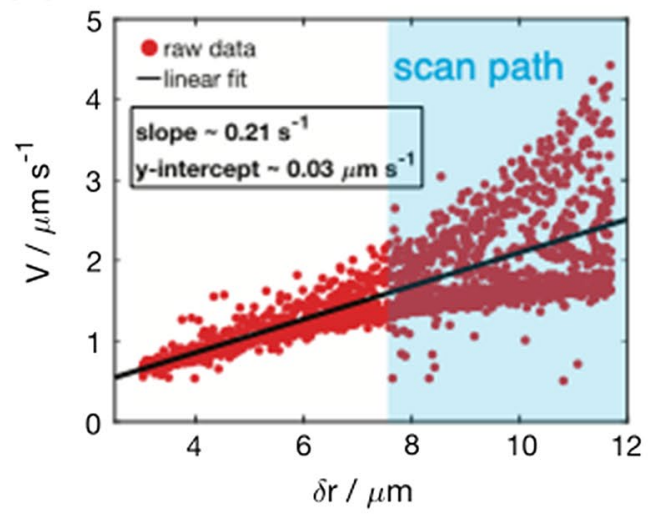

(e)
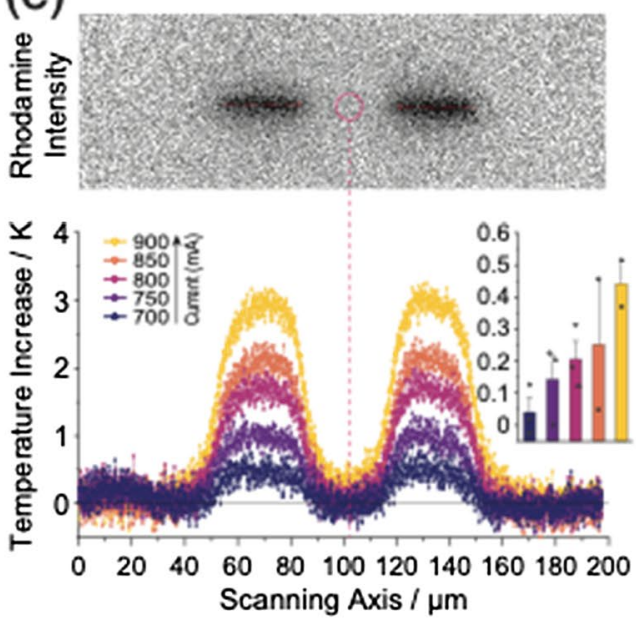

Fig. 1 (See legend on previous page.)

force-extension relationship displayed by our optically induced hydrodynamic trap. The trapping potential generated by optical tweezers is approximately harmonic for small particle displacements, which is typically verified by Stokes' drag calibration [11-13]. We therefore adopted a similar approach to determine the velocity-distance relationship applicable to our system. We displaced a $3-\mu \mathrm{m}$ trapped polystyrene particle away from the stagnation point (Fig. 3a) and then followed its relaxation behaviour for a total of five independent acquisitions (Fig. 3b). 


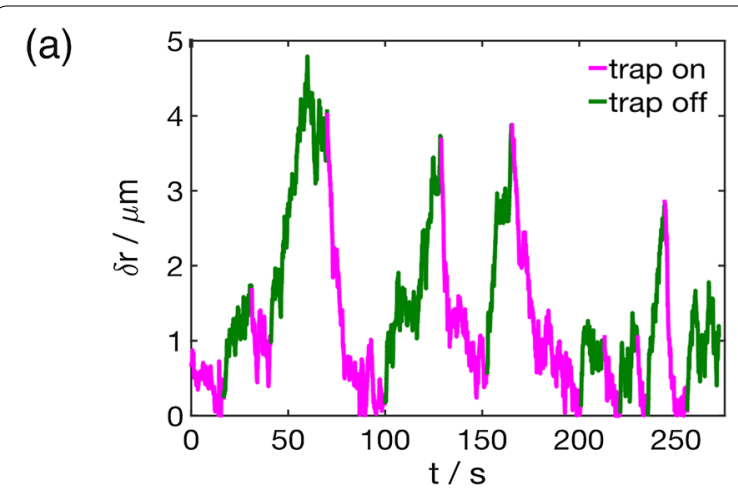

(b)

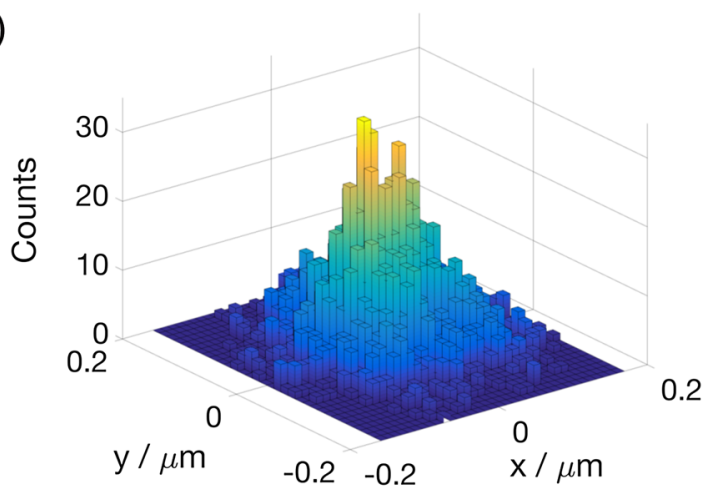

(c)

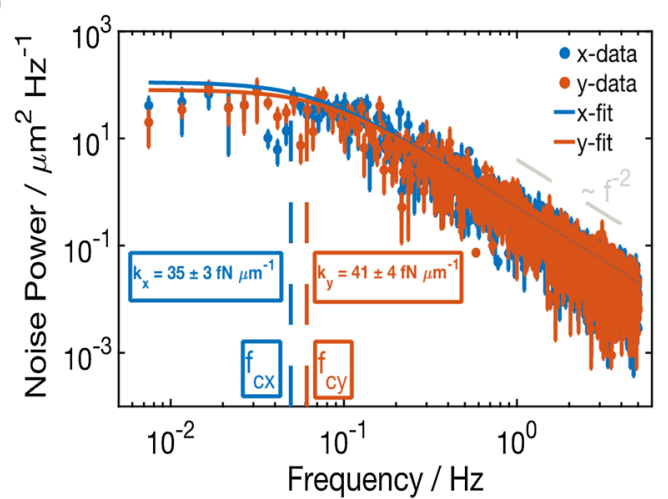

Fig. 2 Optofluidic particle trapping and its unperturbed steady-state dynamics. a Reproducible counterflow trapping (magenta) and free Brownian diffusion (green), illustrating the restoring effect of the thermoviscous trap and its role in limiting the spatial region sampled by the probe. $\mathbf{b}$ A histogram (heatmap) of the phase space explored by the trapped 3- $\mu \mathrm{m}$ particle in deionised water that indirectly shapes the outlines of the trap profile. c Similarly to optical tweezers, power spectral density analysis can be used to obtain a Lorentzian fit to the Fourier-transformed flow-trapping data and thus yield an accurate trap stiffness estimate along each transverse coordinate. The trap symmetry is evident from the agreement between the two in-plane stiffnesses, which overlap within the calculated uncertainties. The errors were calculated following a logarithmic binning procedure, where the magnitude of the standard deviation is inversely related to the number of data points comprising each bin
We observed an exponential approach to the stagnation point (Fig. 3c), suggesting a linear velocity-displacement relationship, where a particle displaced further from the trap is dragged towards the stagnation point at a linearly faster rate. The trapping timescale in optical-tweezer experiments is given by $\tau=6 \pi \eta R / k$ [13], and hence we used the linear fit in Fig. $3 \mathrm{~d}$ to obtain a second estimate of the trap stiffness. The latter agreed closely with our previous PSD roll-off estimate, this time yielding a value as low as $33 \pm 3 \mathrm{fN} / \mu \mathrm{m}$. Given the linear velocity-displacement relationship, these results suggest that our optically induced hydrodynamic trapping approach could be used to measure forces in the femtoNewton range by reading the displacement at which Stokes' forces and external forces balance each other.

\subsection{External force calibration}

To investigate the behaviour of the trap in response to externally applied forces, we used a custom-made electromagnetic needle to exert well-quantified forces on a $5.82-\mu \mathrm{m}$ magnetic particle in deionised water. The MSD analysis at each applied current (Fig. 4a) verified the active magnetic driving of the particle in the absence of any flows through the squared long lag-time dependence of the MSD, indicative of directed superdiffusion. The effect of the enhanced magnetic pulling forces on the bead was also evident at short lag times, where we observed enhanced particle diffusion (see also Additional file 4: Fig. S3). By extracting the long-time (steady-state) velocity $V$ of the bead in each case, we were able to calibrate the magnetic forces (Fig. $4 \mathrm{~b}$ ) through a force balance with a known Stokes' drag force via the relation $F_{S}=-6 \pi \eta R V[14]$.

\subsection{Confirmation of the counterflow trap stiffness through a force balance}

Next, we asked if the apparent spring constant that was indicated by the particle dynamics in the absence of external forces could be confirmed and effectively used to determine the magnitude of the external forces pulling the particle and leading to its displacement. In Fig. 4c (left-hand image), we show a snapshot of the position of a trapped $5.8-\mu \mathrm{m}$ paramagnetic bead. The bead was again observed to explore a very narrow region near the stagnation point formed between the two scan paths. Upon application of a magnetic field, the bead moved away from the stagnation point (right-hand image in Fig. 4c) to a steady-state position in the direction of the magnetic field source. Notably, increasing the magnetic field resulted in larger displacements (Fig. 4d), with an average 
(a)

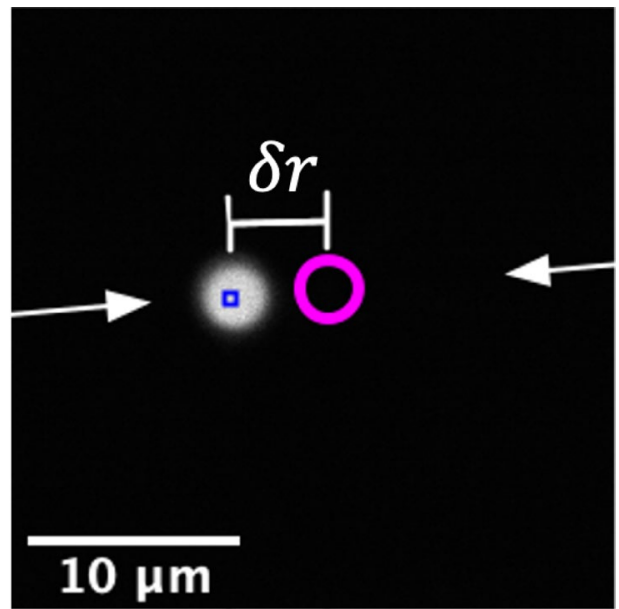

(c)

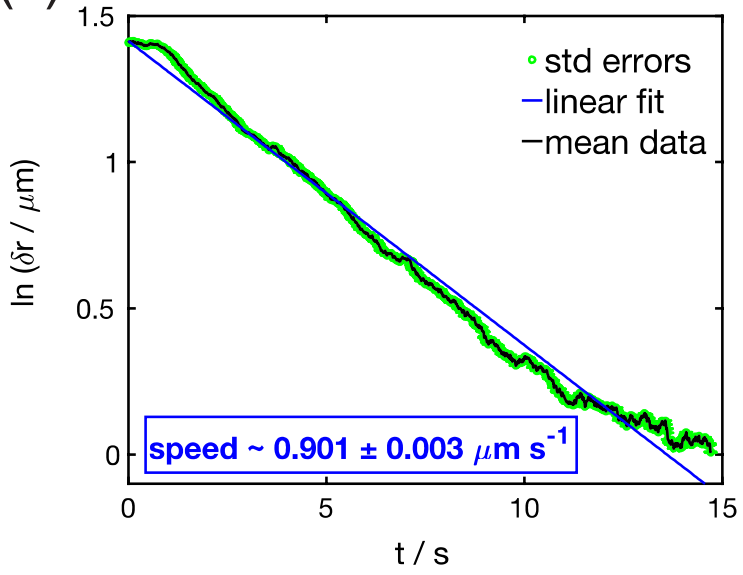

(b)

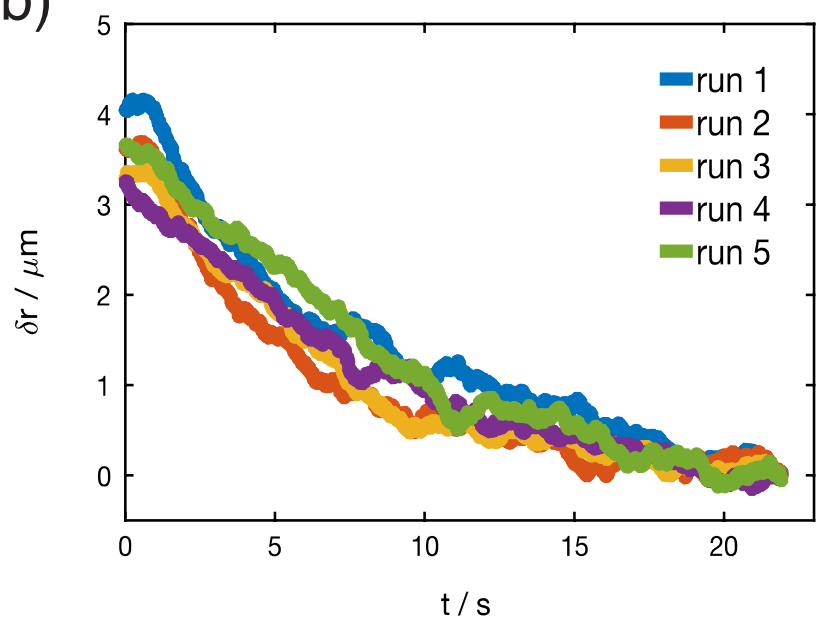

(d)

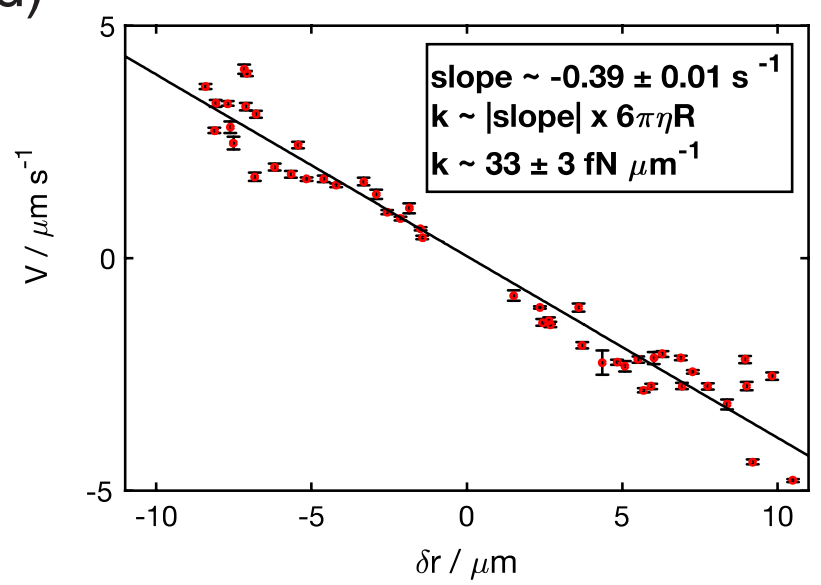

Fig. 3 Quantification of out-of-equilibrium trapping dynamics, linear velocity-displacement relationship and exponential relaxation dynamics following initial perturbation. a Snapshots showing a $3-\mu \mathrm{m}$ particle in water displaced from the stagnation point by an amount $\delta r$, causing the flows to reorient and counteract this displacement (Additional file 3: Video SV3). b Exponential approach of the displaced particle in a 50:50 water:glycerol mixture. $\mathbf{c}$ Plotting the mean of the five acquisitions in $\mathbf{b}$ reveals the linear velocity-displacement relationship near the stagnation point. The shaded region represents the calculated standard deviation. $\mathbf{d}$ Negative linear velocity-displacement relationship near the symmetry axis and close to the stagnation point, extracted from repeatedly performing the analysis in $\mathbf{c}$. The slope provides another estimate of the confinement strength, agreeing closely with the trap stiffness in Fig. 2c. The errors have been calculated via linear regression and the error in the trap stiffness was inferred from the fractional error in the slope

characteristic timescale for reaching steady-state of $\tau \sim$ 13 s (cf. Additional file 4: Fig. S5 for an extended analysis). Bringing the scan lines closer to the bead considerably increases both the confinement and the temporal resolution of the method with sub-second relaxation times (cf. Additional file 4: Fig. S6). By relating the applied current to the calculated magnetic force, we confirmed a linear force-extension relationship (Fig. 4e), which directly demonstrates the ability to sense femtoNewton-range external forces acting on trapped particles. A retrieved spring constant of $35 \pm 4 \mathrm{fN} / \mu \mathrm{m}$ showed close agreement with the previous estimations from steady-state and outof-equilibrium dynamics. Systematic analysis further revealed that trap stiffness scales linearly with bead size (cf. Additional file 4: Fig. S7). The counterflow forces determined by the application of well-controlled magnetic forces could therefore be used to quantify any other externally applied unknown force acting on the particle. 
(a)

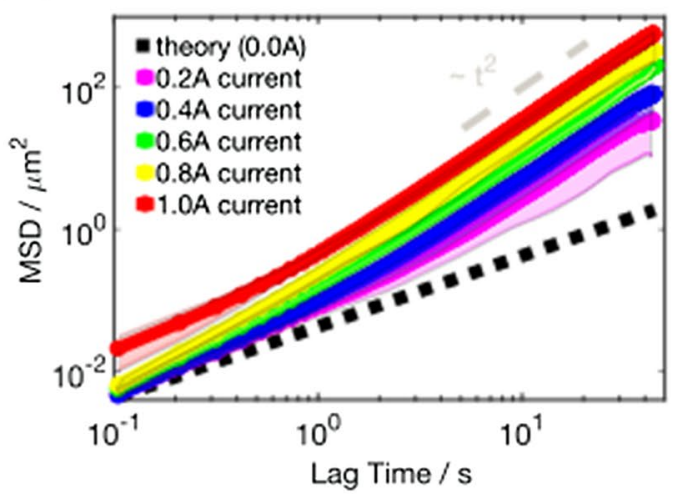

(c)

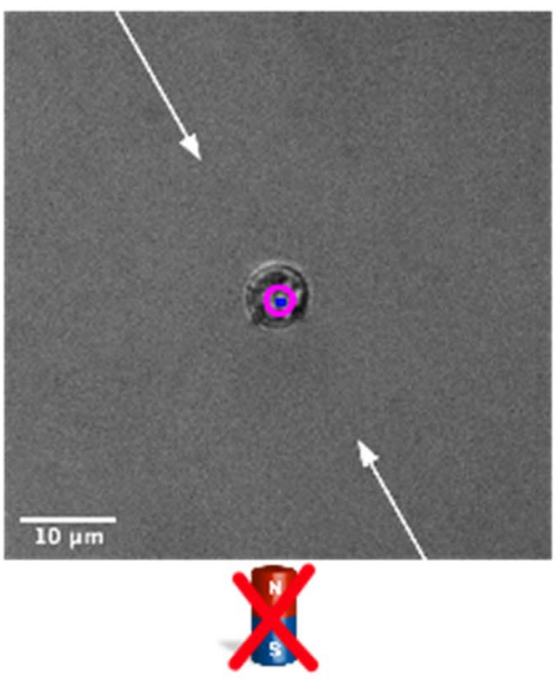

(d)

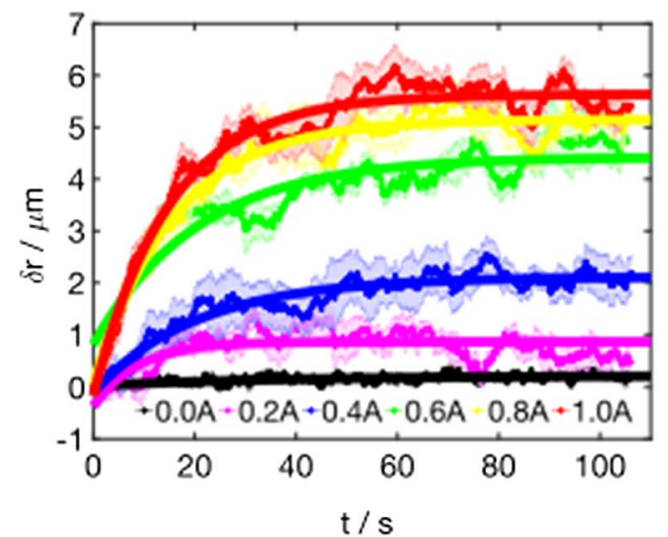

(b)
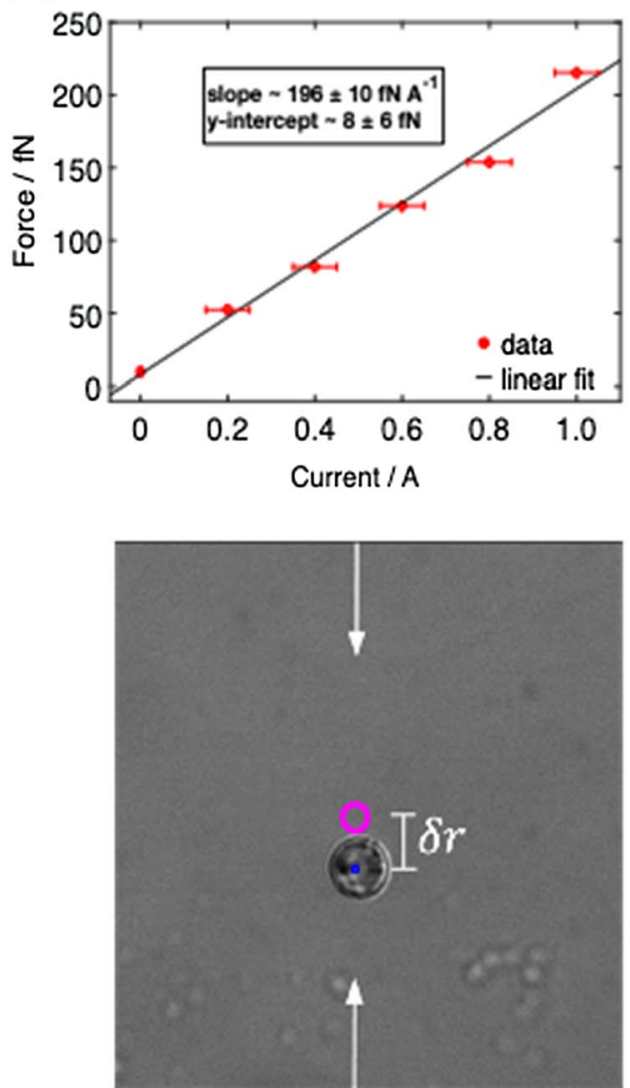

(e)

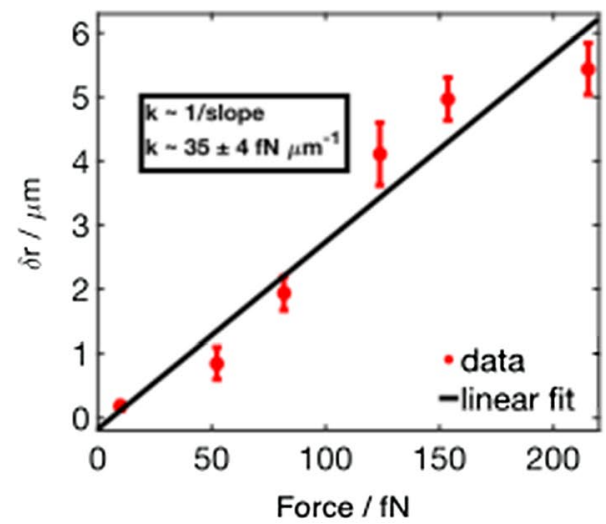

Fig. 4 Linear force-extension relationship enables measuring external forces. a Magnetic bead dynamics undergoing a transition from diffusive (Additional file 4: Fig. S4) to directed motion with increasing, current-controlled external field. b Magnetic force calibration via frictional dissipation. Data averaged over 5 repeats, each lasting ca. $45 \mathrm{~s}$. The errors in the measured forces are contained within the marker size and are negligible compared to the variation in the current used to operate the electromagnetic needle. c Steady-state position of the trapped bead in the absence (left) and presence (right) of an external magnetic force. $\mathbf{d}$ Bead displacement from the stagnation point upon the application of varying magnetic fields. The exponential approach could be readily fitted onto a master curve (Additional file 4: Fig. S5). e Linear force-displacement relationship with a spring constant of $\mathrm{k}=35 \pm 4 \mathrm{fN} / \mu \mathrm{m}$. Shaded regions and error bars in all plots represent standard deviations 


\subsection{Force measurements close to the thermal limit}

The explicit application of an external force confirmed our previous hypothesis and demonstrated the utility of our system as a highly sensitive colloidal sensor of femtoNewton forces. Furthermore, a histogram (Fig. 5a) reflecting the fluctuations of the magnetic particle around its steady-state position, at which the counterflows balance precisely the magnetic force, revealed that the measurements reported here are close to thermally limited. The detection of small forces is accompanied with a wide potential (Fig. 5b) that enables highly sensitive trapping of particles of arbitrary material over an extended spatial range. In addition, the optically generated hydrodynamic trap presented here is highly tunable, enabling further optimisation through adjusting the laser power, the scan path length or the counterflow update rate. Further, optical control capabilities will enable the generation of flow fields with
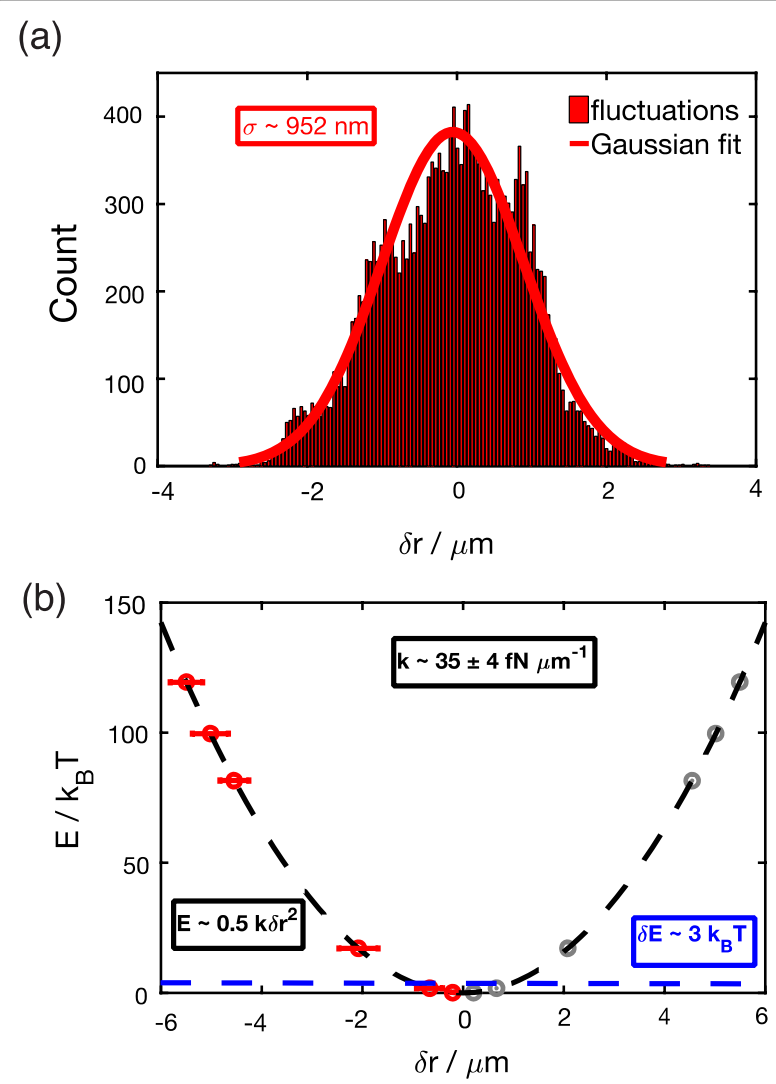

Fig. 5 Force measurements close to the thermal limit. a Histogram of positional fluctuations of a magnetic bead around its field-dependent steady-state position. The reported value corresponds to the standard deviation of the Gaussian fit. b Harmonic potential fitted through the data in Fig. 4e and extrapolated across the stagnation point. The actual measured experimental data are shown in red, while mirrored data are marked in grey. The measured fluctuation in a amounts to ca. 3 times the thermal energy at room temperature even richer topologies and singularities that would be used as attractors or repellors [15].

\section{Conclusions and outlook}

Overall, we describe a highly sensitive, tunable and contact-free trap, generated by two counterdirected optically induced thermoviscous flow fields. The trap is probe-material independent and compatible even with metallic beads. We expect this novel approach to be highly relevant to address the rising concerns regarding non-physiological effects of highly focused radiation on living systems as well as the geometrical and operational limitations of classic microfluidic traps. The arbitrarily defined scan paths and resulting stagnation point render this approach highly localised and flexible. The level of heating due to the induced thermoviscous flows was previously found to be tolerable by in-vivo systems [16], where in the case of the dual flows the region around the stagnation point is heated to an even lower extent (Fig. 1e), which may enable in-vivo applications. In the context of materials science, the method may prove highly suitable for the characterisation of mechanical forces occurring in complex fluids, where the magnitude of the applied flows can be carefully tuned so as not to disrupt the structure of the test sample. Further, our optofluidic trap is an appealing alternative or complement to optical tweezers for probing the active microrheology of weak viscoelastic networks, where the use of a shallow potential minimises instrumental artefacts due to introducing high apparent elasticity. Finally, the ability of the counterflow trap to sense femtoNewton forces on a micrometre scale may prove particularly advantageous in mechanobiological cell signalling assays, provided that challenges related to decoupling the effect of the flows are first overcome.

\section{Materials and methods}

\subsection{Experimental setup}

The experiments reported herein were conducted using the focussed light-induced cytoplasmic streaming (FLUCS) microscope described by Mittasch et al. [16] complemented by novel closed-feedback control scheme implemented in LabVIEW. In brief, acoustooptic scanning (two-axis AOD, AA.DTSXY-A6, Pegasus Optik) of an infrared laser beam (1455 nm wavelength, Raman laser, CRFL-20-1455-OM1, 20 W, Keopsys, used in $\mathrm{CW}$ mode) through a custom-coated $60 \times$ objective lens (UPLSAPO, NA $=1.2, \mathrm{~W}-\mathrm{IR}$ coating, Olympus) was used to generate dynamic heat patterns. The laser scanning along each one of the two paths described in Fig. 1 was performed at $2 \mathrm{kHz}$ scan rate and the scan path positions were updated approximately every $20 \mathrm{~ms}$. The delay 
time between measuring the particle's position and reorientation of the scan trajectories was estimated to be ca. $10 \mathrm{~ms}$.

\subsection{Dye-based temperature measurements}

To measure the temperature increase along and inbetween the scan paths, we made use of the temperature sensitive decrease in quantum efficiency that has been well-described for red spectrum dyes, such as Rhodamine B $[17,18]$. Regarding sample preparation, Rhodamine B solution (Sigma, 02558) was diluted to $10 \% \mathrm{v}$ in water and images were acquired before and during laser application. Using the Calculator Plus function in ImageJ (v. 1.52t), images during laser application were divided by the image before to attain a map of relative intensity changes (Fig. 1e). Using a controlled temperature stage, we measured the relative intensity decrease to be $1.3 \% / \mathrm{K}$. Using this, maps of intensity changes could further be converted into temperature maps, which allows determining the temperature increase on and in-between the scan paths. To exclude thermophoretic effects, which could result in lower dye intensity due to concentration gradients and not due to quantum yield, a fast-spinning wheel $(5 \mathrm{~Hz})$ with partial openings was placed into the laser scan path to discriminate instantaneous intensity changes due to temperature from the slower thermophoretic effects during which gradients are established.

\subsection{Preliminary optofluidic trapping experiments in the absence of other external forces}

We used 3.06- $\mu \mathrm{m}$ spherical polystyrene particles (Fluoresbrite Plain YG 3.0 Microspheres [9003-53-6], Polysciences Inc.) diluted $10^{3} \times$ in deionised water and injected within a $15-\mu \mathrm{m}$ tall chamber made with a plain microscope slide $(76 \times 26 \times 1 \mathrm{~mm}$, Paul Marienfeld $\mathrm{GmbH} \&$ Co. KG, Germany) and a thin round cover slip (22 mm diameter, $170 \mathrm{~m} \mu$ thickness, borosilicate glass). The chamber was sealed with nail polish (speed dry top coat, Essence) to prevent evaporation over the course of the experiments. Imaging was performed with a Zyla 5.5 sCMOS camera and particles tracked using the opensource TrackMate plugin [19] on Fiji. Subsequent analysis involving the calculation of correlation functions was performed on custom-written Matlab routines [7]. Inhouse LabVIEW software combined with custom-written Python routines was used in the trapping experiments to define the scan paths and scan frequency of the infrared laser.

\subsection{Application of an external magnetic force}

The application of an external magnetic force was performed via an in-house custom-made magnetic needle of $4.5 \mathrm{~mm}$ diameter, $100 \mathrm{~mm}$ length and $120^{\circ}$ tip angle.
The material forming the core of the electromagnet was $\mathrm{Hy}-\mathrm{Mu} 80$ (80\% nickel-iron-molybdenum alloy, rod dimensions of $0.380 \times 0.135$ in, National Electronic Alloys Inc., Oakland, NJ), enclosed within a brass frame, following the design of Kollmannsberger and Fabry [20]. However, one notable difference to their work is that we used the needle in conjunction with a closed-chamber design and larger spacing, thus generating a significantly lower effective magnetic field. The dimensions of the brass frame forming the solenoid $(12 \mathrm{~mm}$ in diameter and $37 \mathrm{~mm}$ in length) allowed us to fit about 200 turns of insulated copper wire, which was subsequently connected to a laboratory DC power supply (EA Elektro Automatik, EA-PS 3016-10B). In lieu of using a micromanipulator, the magnetic needle was fixed in place throughout the entire duration of the experiments via a clamp stand with kinematic features, with the stand positioned on the stage so as to translate with it as a unit.

\subsection{Sample preparation for magnetic experiments}

Carboxylated polystyrene-metal spheres (Magnetic Nanoparticles, PMC-5.5, Kisker Biotech GmbH \& Co.) of size $5.82 \mu \mathrm{m}$ were dispersed in deionised water and then injected into an identical $15-\mu \mathrm{m}$ tall chamber to the one described earlier. All experiments were performed at room temperature and at very low particle concentrations to eliminate unwanted hydrodynamic interference due to flow fields set up by particles positioned in proximity to the one being imaged or trapped.

\section{Supplementary Information}

The online version contains supplementary material available at https://doi. org/10.1186/s43593-021-00007-7.

Additional file 1. Determination of the flow fields generated by static counterflows: visualisation of the counterflows using 100-nm polystyrene particles in a viscous honey sample. The counterflows are shown as white arrows, the stagnation point is marked in magenta and some of the nanoparticles are labelled in blue to serve as guide to the eye.

Additional file 2. Trapping achieved via dynamic rotation of the feedback-controlled counterflows: demonstration of the proper operation of the feedback-control loop that rapidly rotates the counterflows (white arrows) and allows stable trapping of the microparticle (blue) at the stagnation point (magenta).

Additional file 3. Positional perturbation of a trapped particle and its subsequent relaxation towards a stagnation point: video analysis of the exponential approach of the particle towards the metastable trap position enables determination of the force-extension relationship characterising the counterflow trap. Additionally, a force balance between the Stokes' drag and the counterflow spring-like forces allows determination of the trap stiffness.

Additional file 4. Supplementary information.

Acknowledgements

We thank lain Patten for valuable discussions on the structure and layout of the manuscript. IDS kindly acknowledges funding from the Life grant by Volkswagen Foundation (Grant No. 92772). We gratefully acknowledge help 
from the mechanical workshop at MPI-CBG for advice on the design and implementation of the electromagnetic needle used in the external force measurements.

\section{Authors' contributions}

IDS and MK conceived the experiments. IDS collected and analysed the data. NM implemented and developed the dual-flow software control used in the data acquisition. BS analysed the flow-field behaviour and temperature effects of the thermoviscous flows while also contributing to the implementation of the magnetic-field experiments. EE provided technical support with operating the setup and contributed to the data acquisition and analysis of the flowfield profile. MK supervised the project. IDS and MK wrote the manuscript. All authors contributed to revisions of the manuscript. All authors read and approved the final manuscript.

\section{Availability of data and materials}

Data available from the corresponding author upon reasonable request.

\section{Declarations}

\section{Competing interests}

IDS, MK and NM are in the process of preparing an application for European patent for technology related to this publication. Further, MK declares an ongoing consultancy relationship with Rapp Optoelectronic $\mathrm{GmbH}$.

\section{Author details}

${ }^{1}$ Max Planck Institute of Molecular Cell Biology and Genetics, Pfotenhauerstraße 108, 01307 Dresden, Germany. ${ }^{2}$ Centre for Systems Biology, Pfotenhauerstraße 108, 01307 Dresden, Germany. ${ }^{3}$ Cluster of Excellence Physics of Life, TU Dresden, Dresden, Germany.

Received: 23 September 2021 Revised: 22 October 2021 Accepted: 23

October 2021

Published online: 08 December 2021

\section{References}

1. E. Erben et al., Feedback-based positioning and diffusion suppression of particles via optical control of thermoviscous flows. Opt. Express 29, 30272-30283 (2021)

2. F.M. Weinert, J.A. Kraus, T. Franosch, D. Braun, Microscale fluid flow induced by thermoviscous expansion along a traveling wave. Phys. Rev. Lett. 100, 164501 (2008)

3. F.M. Weinert, D. Braun, Optically driven fluid flow along arbitrary microscale patterns using thermoviscous expansion. J. Appl. Phys. 104, 104701 (2008)

4. M. Tanyeri, M. Ranka, N. Sittipolkul, C.M. Schroeder, A microfluidicbased hydrodynamic trap: design and implementation. Lab. Chip 11, 1786-1794 (2011)

5. M. Tanyeri, E.M. Johnson-Chavarria, C.M. Schroeder, Hydrodynamic trap for single particles and cells. Appl. Phys. Lett. 96, 224101 (2010)

6. U.G. Būtaitè et al., Indirect optical trapping using light driven micro-rotors for reconfigurable hydrodynamic manipulation. Nat. Commun. 10, 1215 (2019)

7. Stoev, I. D., Caciagli, A., Xing, Z. \& Eiser, E. Using single-beam optical tweezers for the passive microrheology of complex fluids. In: Optical Trapping and Optical Micromanipulation XV vol. 10723 107232D (International Society for Optics and Photonics, 2018).

8. K. Berg-Sørensen, H. Flyvbjerg, Power spectrum analysis for optical tweezers. Rev. Sci. Instrum. 75, 594-612 (2004)

9. N. Malagnino, G. Pesce, A. Sasso, E. Arimondo, Measurements of trapping efficiency and stiffness in optical tweezers. Opt. Commun. 214, 15-24 (2002)

10. A. van der Horst, N.R. Forde, Calibration of dynamic holographic optical tweezers for force measurements on biomaterials. Opt. Express $\mathbf{1 6}$ 20987-21003 (2008)

11. H. Felgner, O. Müller, M. Schliwa, Calibration of light forces in optical tweezers. Appl. Opt. 34, 977-982 (1995)
12. J. Gieseler et al., Optical tweezers — from calibration to applications: a tutorial. Adv. Opt. Photonics 13, 74-241 (2021)

13. P.H. Jones, O.M. Maragò, G. Volpe, Optical Tweezers: Principles and Applications (Cambridge University Press, Cambridge, 2015)

14. J.C. Loudet, P. Hanusse, P. Poulin, Stokes drag on a sphere in a nematic liquid crystal. Science 306, 1525-1525 (2004)

15. L. Giomi, Ž Kos, M. Ravnik, A. Sengupta, Cross-talk between topological defects in different fields revealed by nematic microfluidics. Proc. Natl. Acad. Sci. 114, E5771-E5777 (2017)

16. M. Mittasch et al., Non-invasive perturbations of intracellular flow reveal physical principles of cell organization. Nat. Cell Biol. 20, 344-351 (2018)

17. S.M. Hirsch et al., FLIRT: fast local infrared thermogenetics for subcellular control of protein function. Nat. Methods 15, 921-923 (2018)

18. A. Singhal, S. Shaham, Infrared laser-induced gene expression for tracking development and function of single C. elegans embryonic neurons. Nat. Commun. 8, 1-3 (2017)

19. J.-Y. Tinevez et al., TrackMate: an open and extensible platform for singleparticle tracking. Methods 115, 80-90 (2017)

20. P. Kollmannsberger, B. Fabry, BaHigh-force magnetic tweezers with force feedback for biological applications. Rev. Sci. Instrum. 78, 114301 (2007) 